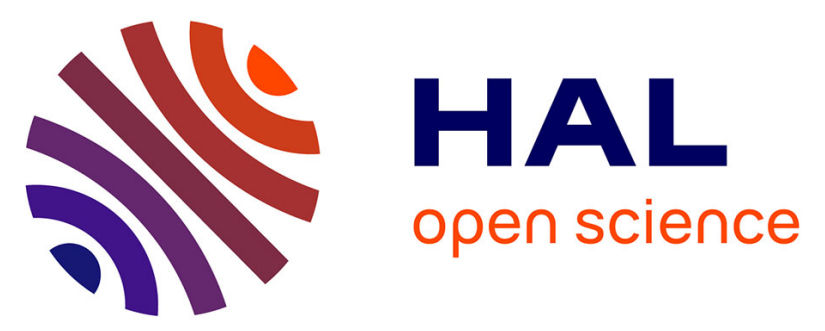

\title{
Oscillation regimes produced by an alto saxophone: Influence of the control parameters and the bore inharmonicity
}

\author{
Jean-Baptiste Doc, Christophe Vergez
}

\section{- To cite this version:}

Jean-Baptiste Doc, Christophe Vergez. Oscillation regimes produced by an alto saxophone: Influence of the control parameters and the bore inharmonicity. Journal of the Acoustical Society of America, 2015, 137 (4), pp.1756. 10.1121/1.4916197 . hal-01229842

HAL Id: hal-01229842

https://hal.science/hal-01229842

Submitted on 17 Nov 2015

HAL is a multi-disciplinary open access archive for the deposit and dissemination of scientific research documents, whether they are published or not. The documents may come from teaching and research institutions in France or abroad, or from public or private research centers.
L'archive ouverte pluridisciplinaire HAL, est destinée au dépôt et à la diffusion de documents scientifiques de niveau recherche, publiés ou non, émanant des établissements d'enseignement et de recherche français ou étrangers, des laboratoires publics ou privés. 


\title{
Oscillation regimes produced by an alto saxophone : influence of the control parameters and the bore inharmonicity.
}

\author{
J.-B. Doc ${ }^{1}$ and C. Vergez ${ }^{1}$ \\ ${ }^{1}$ LMA, CNRS, UPR 7051, Aix-Marseille Univ, Centrale Marseille, F-13402 Marseille Cedex 20, France
}

The aim of this work is to highlight experimentally how inharmonicity of the bore resonance frequencies of an alto saxophone influence the nature of the oscillation regimes. A variable volume branching from the neck of an alto sax at an appropriate position allows to change the frequency of the first resonance independently from the second. A blowing machine with artificial lips is used to make the saxophone play while controlling independently the control parameters : the blowing pressure and an embouchure parameter. Values of these parameters are estimated experimentally through the measurement of the nonlinear characteristics linking the mean air flow blown into the instrument to the static pressure difference across the reed. Experiments with different values of the control parameters as well as of the inharmonicity produce different kinds of oscillation regimes. These regimes are categorized through the analysis of the pressure signal inside the mouthpiece. The resulting maps demonstrate that the emergence of quasi-periodic regimes, and their extent, depend on the level of inharmonicity, but also on the values of the control parameters. Periodic regimes playable by choosing appropriate values of the control parameters also differ according to the level of inharmonicity, a higher inharmonicity facilitating the emergence of the third register.

\section{Introduction}

The construction of musical wind instruments requires, for example, drilling side holes and adding a mouthpiece and bell. This may alter the harmonicity of the instrument, which means that the different resonance frequencies deviate from a perfect harmonic series. Inharmonicity of resonance frequencies has a direct influence on the intonation of a musical wind instruments ${ }^{1}$, as well as on the timbre of the sounds produced. Moreover, the inharmonicity of a wind instrument may trigger the production of multiphonics $^{2}$, which correspond to a quasi-periodic oscillating regime $^{3}$. Therefore, inharmonicity appears as a key factor to consider when designing musical wind instruments.

Multiphonics can be easily obtained on wind instruments played unconventionally, for instance using fork fingerings or a modified embouchure. These multiphonics are perceived as chords or rolling or beating sounds ${ }^{4}$ and are commonly used in Jazz or contemporary music. However, even when the instrument is played in a conventional way, undesirable quasi-periodic regimes may be produced. In that case, craftsmen obviously try to avoid this type of behavior which affects the playability of the instrument.

Quasi-periodic regimes have already been studied for organ pipes and flutes ${ }^{5 ; 6 ; 7}$ or reed instruments $8 ; 9$. However the emergence of quasi-periodic regimes in musical instruments is still a poorly understood phenomenon. For reed instruments, the paper by Dalmont et al. ${ }^{10}$ presents the first experimental evidence that the harmonicity of the resonances may influence the production of quasi-periodic regimes by an alto saxophone. This pioneering work has recently been revisited in a study ${ }^{11}$ based on the use of a numerical model of the sound production. This study shows that control parameters set by the player have also a significant influence on the production of quasi-periodic regimes.
The goal of this paper is to highlight experimentally the influence of the harmonicity of the resonances on the sound production by an alto saxophone. The analysis focuses on regimes produced, and questions relative to timbre are ignored. The experiment from Dalmont et al. ${ }^{10}$ is carried out again : a piston is added perpendicularly to the neck of the saxophone (see fig. 2(b)) at a location which allows to alter the resonance frequency of the lowest mode without affecting the resonance frequency of the second mode. The inharmonicity of the lowest modes of the instrument can consequently be controlled by the experimenter.

An artificial mouth is used in order to make the saxophone play with two control parameters : the embouchure settings and the blowing pressure (considered independent for this study). These parameters are estimated experimentally according to a model of the acoustical exciter. Sound is recorded for sets of control parameter values, and the nature of the regime is identified through analysis of the signal. These can be represented graphically in maps that allow an easier reading of the experimental data.

Section introduces the experimental device as well as methods used to characterize it. In section , signal processing methods are then presented. Finally, in section, experimental results are presented.

\section{Characterization of the experimental device}

\section{A. Experimental device}

The saxophone used for the experiment is a student model (Yamaha YAS-280), played with a Plasticover reed (force 3) placed on a standard mouthpiece (Yamaha $4 \mathrm{C})$. In order to perform different fingerings, cork pieces are inserted below the corresponding key mechanisms so that the desired keys are maintained closed. Tensioners are also used to keep certain keys closed.

A photo of the experimental device is shown in Figure 1, where the saxophone is laid horizontally in 


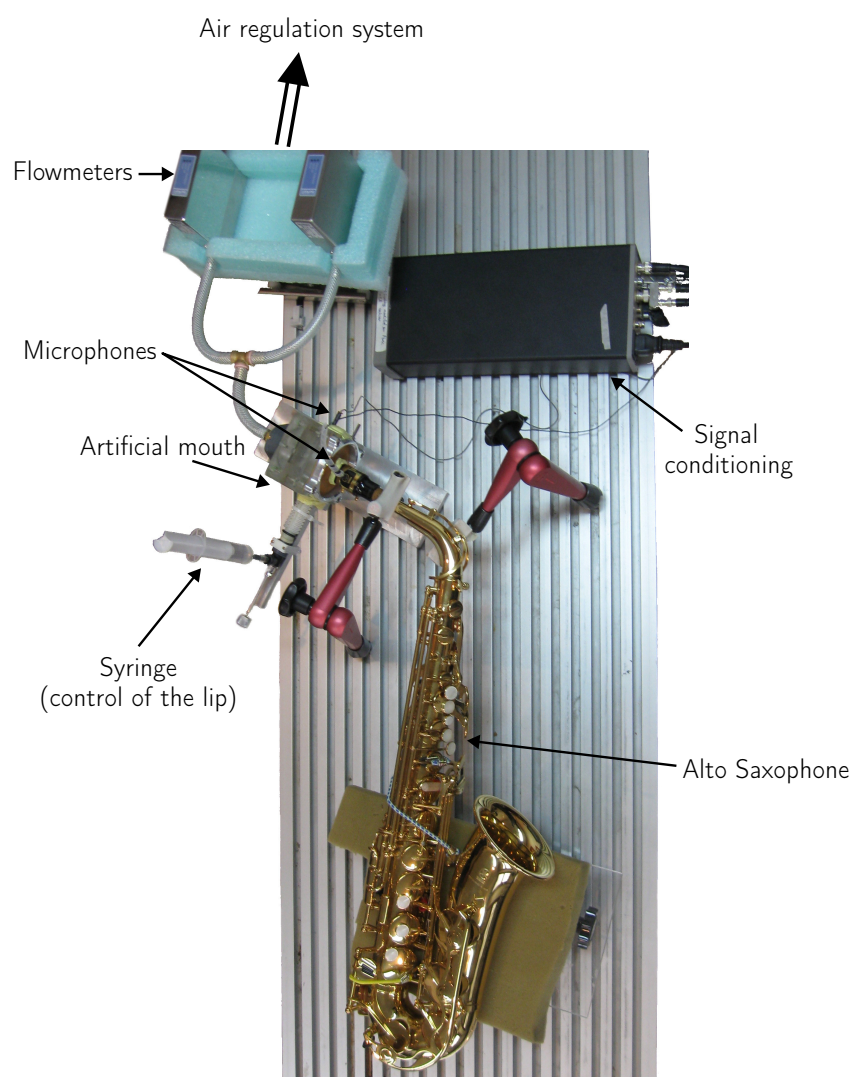

Fig. 1 - Experimental device (top view).

order to be connected to the artificial mouth. The latter is based on a plexiglass box, the inner volume of which is $15 \mathrm{~cm}^{3}$. The control of air pressure inside the box is carried out through a closed-loop. A real-time dSpace controller drives a servo-valve (placed upstream an air tank) according to the static pressure measured in the box. Specific developments have been necessary to achieve a precise control through time of the pressure in the artificial mouth (technical details are available in $\left.{ }^{13}\right)$. Pressure ramps can therefore be generated in the artificial mouth.

Figure 2(a) displays a sketch of the artificial mouth. The mouthpiece of the instrument is connected to the artificial mouth through a rubber seal which ensures air tightness. An artificial lip is pressed against the reed and brings the required damping to avoid squeaks when the instrument is playing. The artificial lip is made with latex tube filled with water through a syringe. Rising the pressure of water increases the bearing force of the lip on the reed, which results in a decreased reed channel opening. The artificial lip is placed around $1 \mathrm{~cm}$ downstream the tip of the reed (see Figure 2(a)).

The static pressure inside the artificial mouth as well as the pressure inside the mouthpiece are measured with Endevco pressure sensors (8507C-5 model). A Burkert flowmeter (model 8701) is used to measure the air volume entering the instrument. A National Instrument acquisition module (NI9215 model) digitizes the experimental signals with a sampling frequency of $44 \mathrm{kHz}$, further recorded on a computer.

\section{B. Characterization of the exciter}

The mouthpiece and the reed together can be seen as an exciter. According to classical textbooks ${ }^{14}$, this

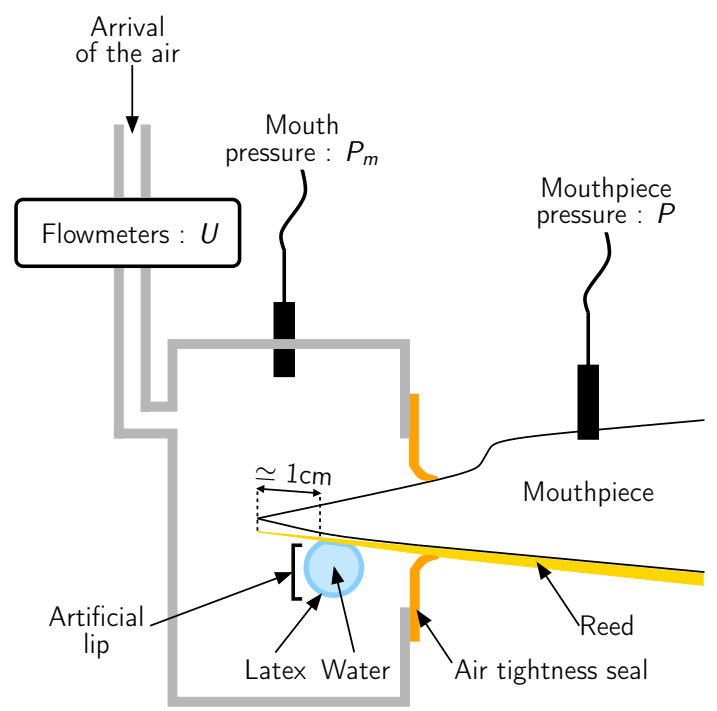

(a)

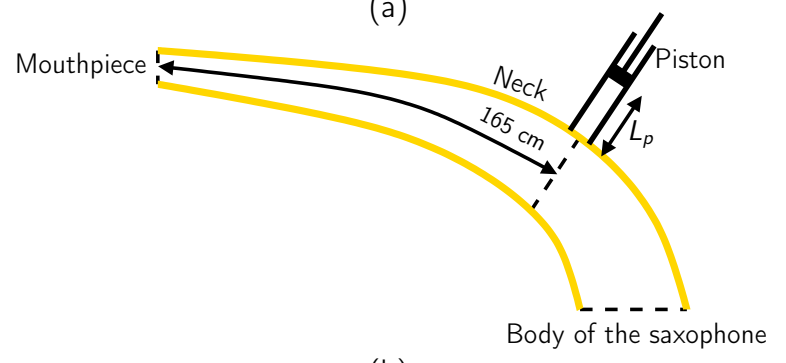

(b)

Fig. 2 - (a) Sketch of the artificial mouth and (b) schematic of the modified saxophone neck. A piston is plugged at $165 \mathrm{~mm}$ from the input of the neck. The inner diameter of the piston is $12 \mathrm{~mm}$.

system can be characterized through the estimation of two physical quantities : the airflow $U$ through the reed channel and the difference $\Delta P$ between the mouth pressure $P_{m}$ and the mouthpiece pressure $P$. The airflow produced by the reed displacement is ignored in this description. The so-called nonlinear characteristics of the exciter $^{15}$ (noted NLC hereafter), connecting these two quantities can be written :

$$
\begin{cases}U=\frac{\zeta}{Z_{c}}\left(P_{M}-\Delta P\right) \sqrt{\frac{|\Delta P|}{P_{M}}} \operatorname{sgn}(\Delta P) \\ \text { if } \Delta P<P_{M}, \\ U=0 & \text { if } \Delta P>P_{M},\end{cases}
$$

where $P_{M}$ is the beating reed pressure, i.e. the minimum pressure difference across the reed to close the reed in the static regime, and $\zeta$ the embouchure parameter defined by :

$$
\zeta=Z_{c} S \sqrt{\frac{2}{\rho P_{M}}},
$$

with $Z_{c}$ the characteristic impedance of plane waves inside the resonator, $S$ the cross-section of the instrument input and $\rho$ the density of air.

Parameters $P_{M}$ and $\zeta$ are estimated experimentally by fitting Eq.(1) onto a measured NLC. Figure 3 displays an example for a given setting of the artificial mouth. Thanks to the measurement, the maximum airflow $U_{\max }$ and the corresponding pressure difference $P_{\max }$ can be estimated. Considering that $P_{M}=3 P_{\max }$, the embouchure 


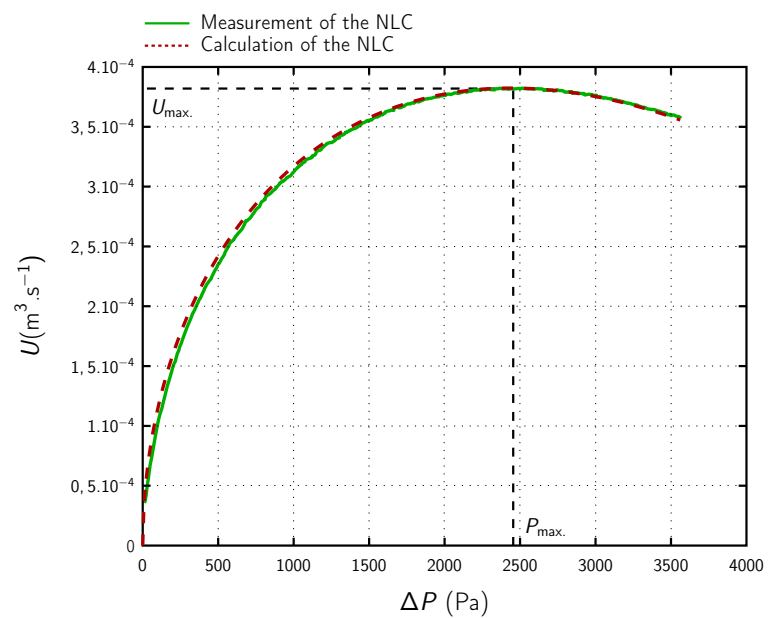

Fig. 3 - (Color online) Measurement of the nonlinear characteristics (NLC) on an alto saxophone (G' fingering) for a given setting of the artificial mouth ( $\zeta \simeq 0.45$ and $P_{M}=7350 \mathrm{~Pa}$ ) and comparison with the fitted model. (Eq. (1)).

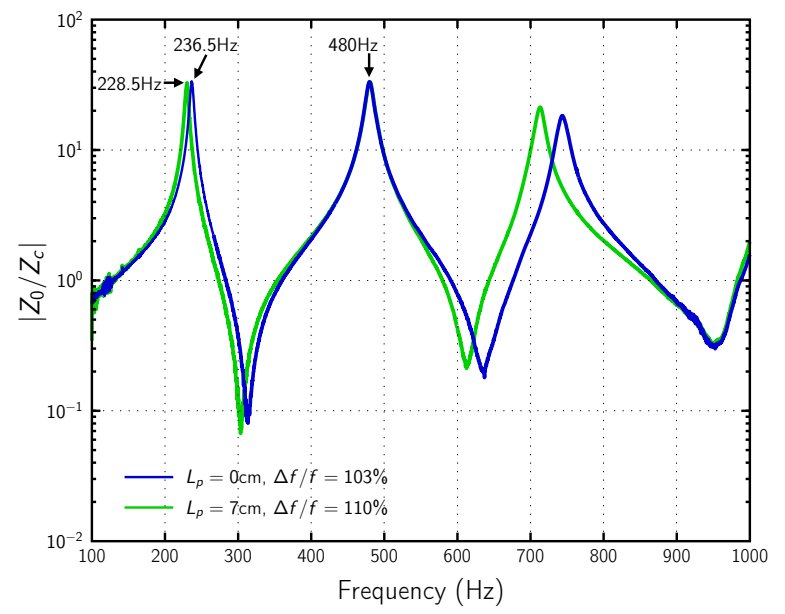

Fig. 4 - (Color online) Modulus of the dimensionless input impedance of the alto saxophone ( $\mathrm{G}^{\prime}$ fingering) for two lengths $L_{p}$.

parameter $\zeta$ is then obtained through the relation ${ }^{15}$ :

$$
\zeta=\frac{\sqrt{3}}{2} \frac{U_{\max }}{P_{\max }} Z_{c} .
$$

The NLC modeled through equation (1) is then plotted using the estimated values of $P_{M}$ and $\zeta$. As shown in Figure 3, the measured and the modeled NLC are nearly identical. Moreover, knowing $P_{M}$ allows to evaluate the dimensionless blowing pressure $\gamma$, defined as the ratio between the mouth pressure and the reed beating pressure : $\gamma=P_{m} / P_{M}$.

For each experiment or for each change in the setting of the experimental mouth, an estimation of $\zeta$ and $\gamma$ parameters is carried out. This allows us to plot the analysis results of the mouthpiece pressure (the different oscillation regimes as explained in section ) on a map representing the control parameters $\zeta$ and $\gamma$.

\section{Characterization of the resonator}

The impedance of the alto saxophone is measured with an impedance sensor ${ }^{16}$ for each fingerings used in the experiment. A cylinder of the same volume as the mouthpiece is placed upstream the instrument. For this study the fingering corresponding to the $\mathrm{G}$ note (as read on a score) of the first register (noted hereafter $G^{\prime}$ ) is used. A piston is soldered to the neck of the saxophone without affecting the inner geometry of the instrument (see Figure 2(b)). For the G' fingering, this allows to alter the first resonance frequency of the instrument without affecting the other resonances ${ }^{10}$. Therefore, pushing/pulling the piston allows to directly modify the inharmonicity of the instrument. In the following, since the amplitudes of the first two peaks are at least twice as large as those of the upper peaks, we focus on the inharmonicity of the two lowest resonance frequencies $f_{1}$ and $f_{2}$ defined by $\Delta f / f=\left(f_{2}-f_{1}\right) / f_{1}$. This quantity will be given as a percentage.

Figure 4 presents the dimensionless input impedance of the alto saxophone for the two extreme positions of the piston. When the piston is completely pushed $\left(L_{p}=0\right)$, there is no modification of the air column added and the resonance frequencies are nearly harmonic $\Delta f / f=103 \%$. When the piston is completely pulled $\left(L_{p}=7 \mathrm{~cm}\right)$, the volume added to the air column induces an inharmonicity so that $\Delta f / f=110 \%$. The input impedance has been measured for different intermediate positions of the piston in order to evaluate the inharmonicity as a function of $L_{p}$.

\section{Data processing}

Measurement and analysis of the acoustic pressure inside the mouthpiece of the instrument allows classifying the different oscillation regimes produced. This classification relies on the identification of the instantaneous frequencies and amplitudes in the recorded signals, as explained above.

\section{A. Instantaneous frequencies and amplitudes of the mouthpiece pressure}

A spectrogram is first calculated according to the mouthpiece pressure signal. From this, the evolution of instantaneous frequencies and amplitudes are estimated depending on the experimental time-varying parameters $\left(\gamma, L_{p}\right.$ or $\left.\zeta\right)$. Practically, the Matlab function findpeaks is used. An additional constraint is imposed on the amplitude of peaks to identified. Thus, only one instantaneous frequency by partial is detected.

Figure 5 displays an example. Dotted lines represent the estimated instantaneous frequencies. The main components of the signal are correctly localized on the spectrogram.

\section{B. Classification of the oscillation regimes}

An algorithm has been developed to classify the different oscillation regimes according to the instantaneous and amplitude frequencies on each frame of the spectrogram. Three types of oscillation regimes are identified : first of all a static regime (no sound), then a quasi-periodic regime (composed of two incommensurate frequencies) and finally a periodic regime (obviously different playing frequencies are possible). In practice, the 

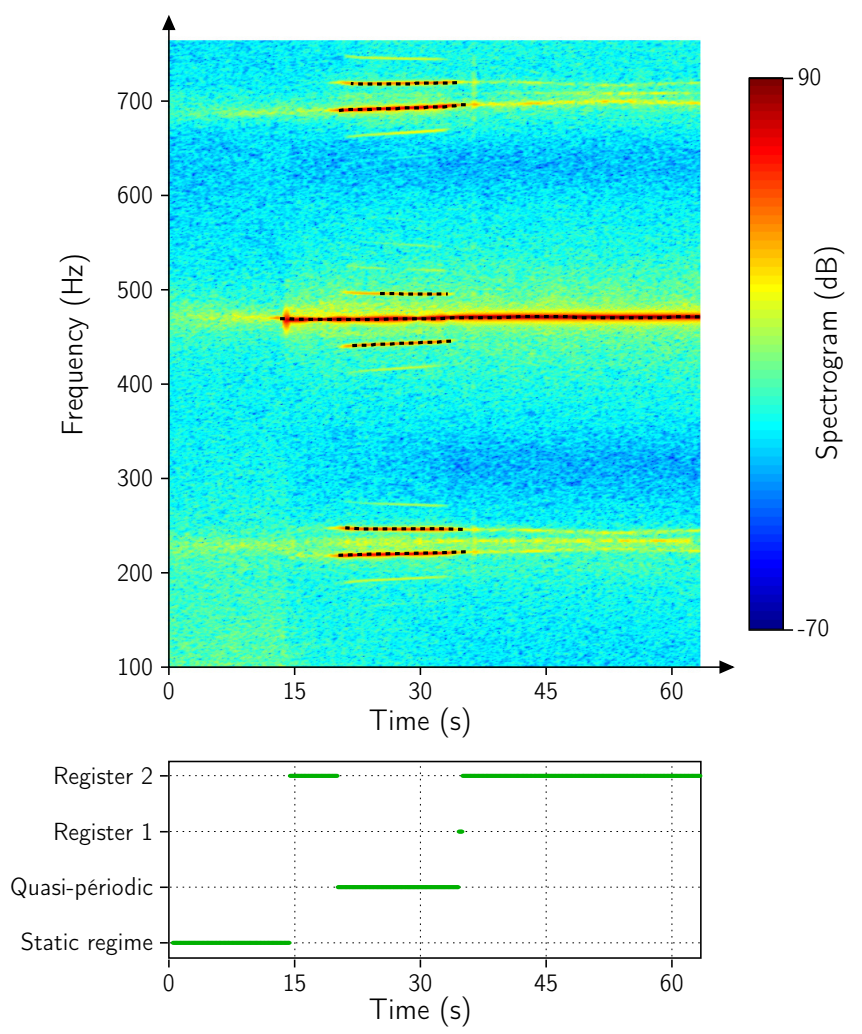

Fig. 5 - (Color online) Top : spectrogram of a pressure signal recorded in the mouthpiece for a linearly increasing blowing pressure. Estimated instantaneous frequencies are plotted in dashed lines. Bottom : oscillating regimes labeled using the algorithm developed based on the instantaneous frequencies.

instrument does not produce the fourth register. That is why the identification is limited in frequency up to $1000 \mathrm{~Hz}$ (frequency lower to the one of the 4th harmonic).

The main steps of the algorithm are constructed following this hierarchical order :

- If no instantaneous frequency is detected or if no instantaneous amplitude crosses a user-defined threshold : the regime is classified as static.

- If more than one instantaneous frequency is detected, ratios between each pair of consecutive frequencies are calculated. If at least one of these ratios belongs to the interval $[0.7,1.3]$, the oscillation regime is classified as quasi-periodic. We can note that the method is configured to calculate a only one instantaneous frequency by harmonic component.

- Otherwise the smallest instantaneous frequency is compared to the frequencies of the expected notes for the fingering considered (mostly the different harmonics of low $\mathrm{G}$ ). If there is match within $5 \%$, the oscillation regime is classified as periodic.

Figure 5 shows an example of this algorithm output. In this example, a linear increasing ramp of the blowing pressure is carried out, starting below the oscillation threshold. Hence, a static regime is identified during the first $15 \mathrm{~s}$ of the signal. During the time interval $t=$ $[20,35]$ (s), many non-harmonic frequency components appear on the spectrogram, leading to a classification as (a)

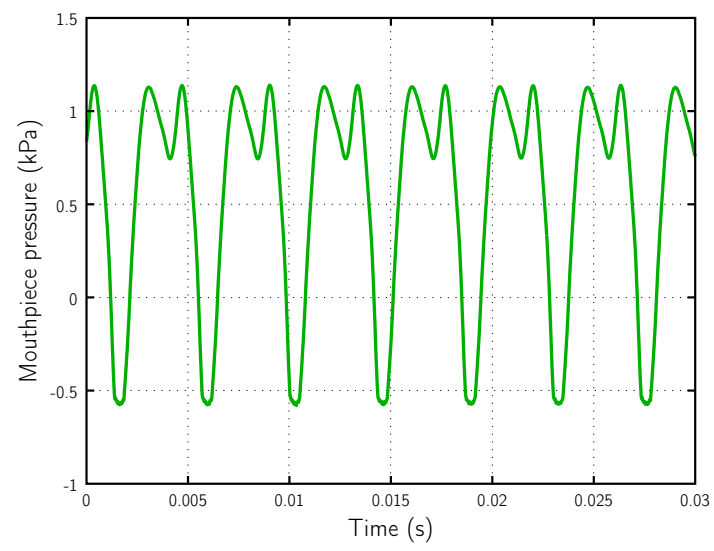

(b)

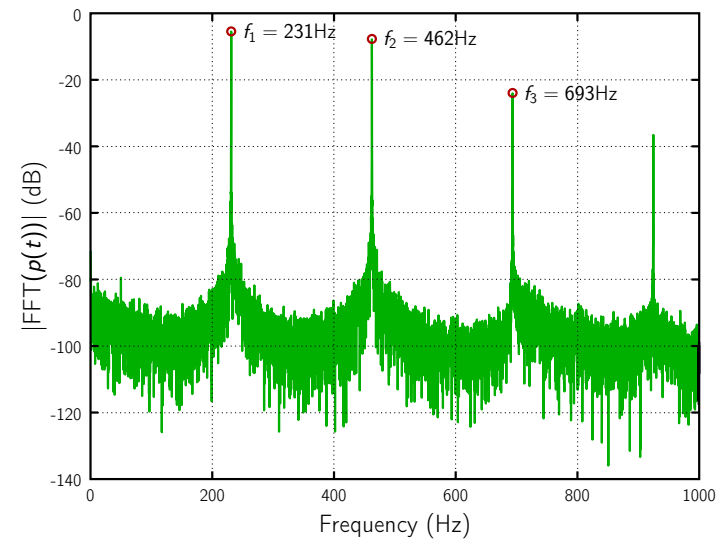

Fig. 6 - (a) Periodic signal in the time domain and (b) spectrum of the mouthpiece pressure ( $\mathrm{G}^{\prime}$ fingering, $\gamma=0.45, \zeta=0.55$ and $\Delta f / f=103 \%)$.

a quasi-periodic regime. The rest of the time, a periodic regime is detected and identified as the second register of the instrument (playing frequency $\simeq 460 \mathrm{~Hz}$ ), which is correct since the lowest resonance frequency of the saxophone is located around $230 \mathrm{~Hz}$ (see Figure 4).

However, due to the choice of time step in the spectrogram, errors may occur during transitions between regimes. Indeed, as seen in Figure 5 around $t=35 \mathrm{~s}$, register 1 is detected on a few consecutive frames. Inspection by eye of the spectrogram reveals that this is a limitation of the algorithm. However this does not hinder the global reading of the output of the algorithm, i.e., the various regimes produced by the instrument.

\section{Experimental results}

\section{A. Nature of the oscillating regimes}

In order to illustrate the various types of oscillation regimes studied below, two examples of periodic and quasi-periodic signals recorded inside the mouthpiece of the saxophone are presented here. Both of these signals correspond to notes played by the artificial mouth with a constant blowing pressure.

Figure 6(a) displays a periodic pressure signal. Inspecting the waveform reveals the classical Helmholtz motion for saxophones ${ }^{18}$, while the spectrum confirms the periodicity of the regime with a playing frequency $f_{1}=231 \mathrm{~Hz}$ (see Figure 6(b)).

Figure 7(a) displays a quasi-periodic regime. The time interval of the figure is large which highlights a 

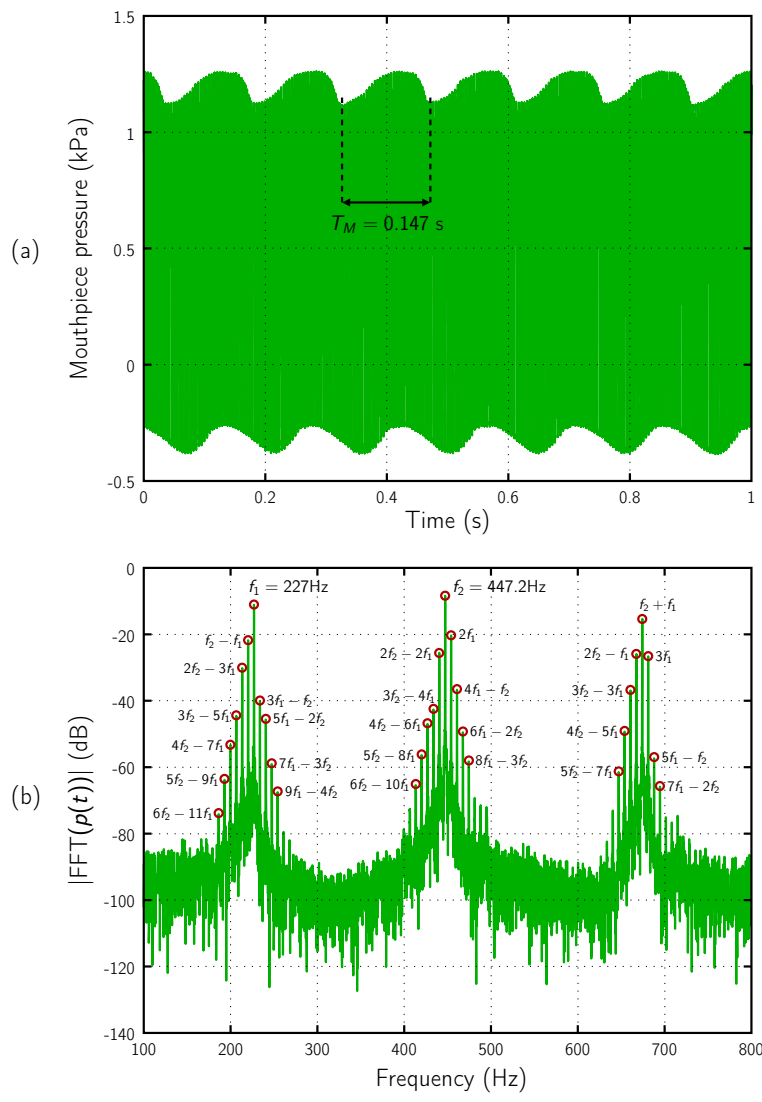

Fig. 7 - (a) Quasi-periodic signal in the time domain and (b) spectrum of the mouthpiece pressure (G' fingering, $\gamma=0.41, \zeta=0.59$ and $\Delta f / f=110 \%)$.

low frequency modulation (around $f_{M}=6.8 \mathrm{~Hz}$ ) of the signal. The corresponding spectrum is shown in Figure 7(b). The envelope modulation in the time domain corresponds to the many spectral components whose frequencies are linear combinations of two inharmonically related frequencies $f_{1}$ and $f_{2}$. Indeed the frequency $f$ of each peak in Figure 7 (b) can be written as $f=n f_{1}+m f_{2}$, where $m, n \in \mathbb{Z}$. Two consecutive peaks are always separated by the same frequency $f_{M}=2 f_{1}-f_{2}$. It is worth noting that $f_{1}$ is close to the lowest resonance frequency of the saxophone for the $G$ ' fingering (see Figure 4). The second frequency $f_{2}$ is not directly linked to the second resonance frequency of the saxophone, and is such that the ratio $f_{2} / f_{1}$ is not an integer.

\section{B. Maps of the oscillation regimes in the control parameters space}

In this section, maps of oscillating regimes (in the control parameter plane $(\zeta, \gamma)$ ) are obtained for different inharmonicity values of the instrument. As it has been previously shown ${ }^{12}$, setting up maps according to various control parameters has the advantage of easing the reading of experimental data. In this study, the measurement of the nonlinear characteristics allows to estimate dimensionless control parameters. This facilitates a better understanding of the range of the control parameters imposed during experimentations.

With the available experimental device, both $\zeta$ and $L_{p}$ have to be varied manually (see section ). On the other

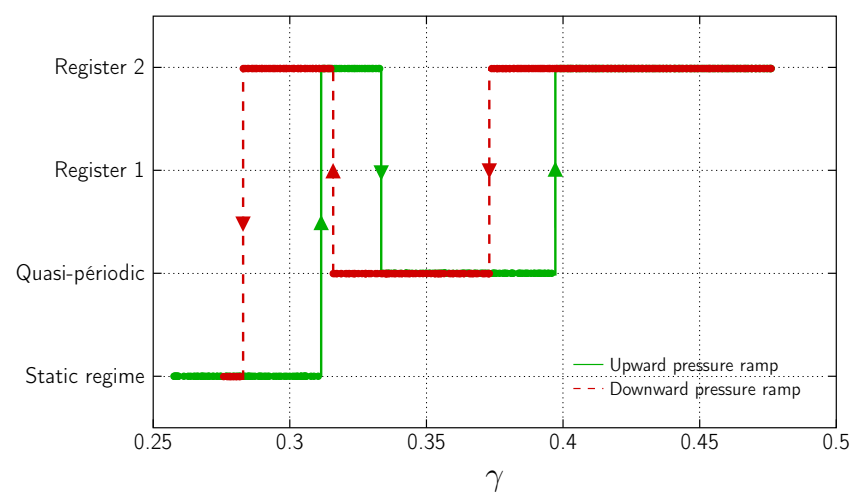

Fig. 8 - (Color online) Example of identification of the oscillating regimes for a given setting of the experimental device ( $\mathrm{G}^{\prime}$ fingering, $\Delta f / f=110 \%$, $\zeta=0.31)$. Linearly increasing (continuous line) and decreasing (dashed line) blowing pressure.

hand, the blowing pressure and its variation through time are precisely controlled through a PID loop ${ }^{13}$. Therefore, to save time and keep a reasonable number of recordings, experiments are carried out with linearly varying blowing pressure : increasing and decreasing ramps, 60s each. These ramps always begin below the oscillation threshold $(\gamma \simeq 0.25)$ and last until the oscillation regime is not willing to change again $(\gamma \simeq 0.5)$. Each time the setting of the artificial mouth is modified, parameters $\zeta$ and $P_{M}$ are estimated through the measurement of the NLC (see section ).

Since measurements are carried out with pressure ramps, thresholds at which the various regimes emerge are different when the ramp is increasing or decreasing. This hysteresis is clearly visible in Figure 8 where an example is displayed, for a given setting of the artificial mouth $(\Delta f / f=110 \%$ and $\zeta=0.31)$. However, since the speed of variation of the blowing pressure is low enough $( \pm 25 \mathrm{~Pa} / \mathrm{s})$, the same evolution of oscillation regimes is produced. Since the precise estimation of the bifurcation thresholds is not of interest in this study and for sake of readability, only the analysis corresponding to increasing ramps will be shown below.

Figure 9 displays different maps in the $(\zeta, \gamma)$ space obtained for various inharmonicities. A periodic regime based on the $n^{\text {th }}$ register of the instrument is noted $\mathrm{R} n$, while QP stands for a quasi-periodic regime. Each oscillation regime is tagged with its own color. Each horizontal line corresponds to the output of the identification algorithm (see Part. ). The static (non-oscillating) regime is not plotted for readability. It is worth noting that results lie on a restricted area of the map, since outside this area, no regime change has been observed.

These maps highlight the influence of inharmonicity with respect to the control parameters $(\zeta$ and $\gamma)$. Recall that, the blowing parameter $\gamma$ is directly linked with the breath pressure. The embouchure parameter $\zeta$ is related to the maximum flow through the reed channel. It mainly depends on the geometry of the mouthpiece, the reed mechanical properties, as well as the player's lip force and position on the reed that control the opening. A "tight embouchure" can be viewed as a low value of $\zeta$ and a "relaxed embouchure" can be viewed as a high value of $\zeta$. 


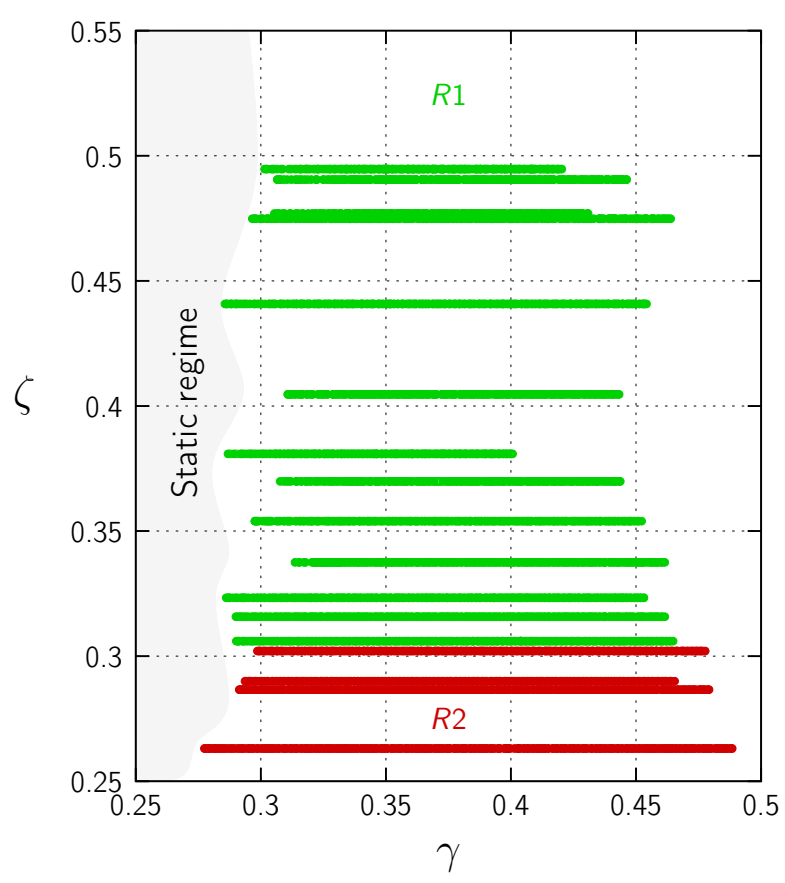

(a)

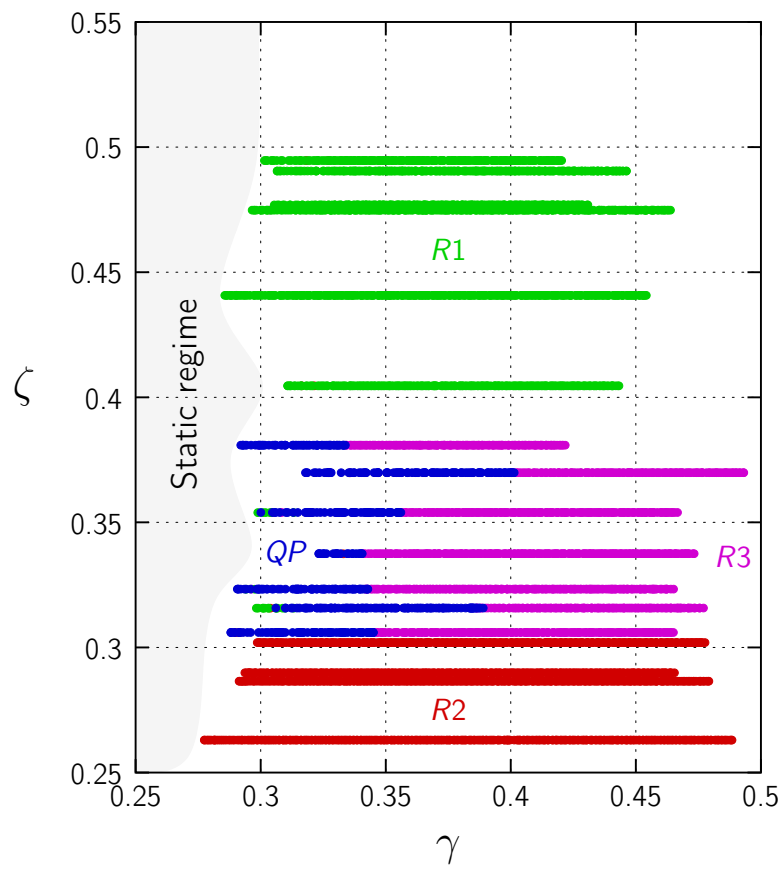

(c)

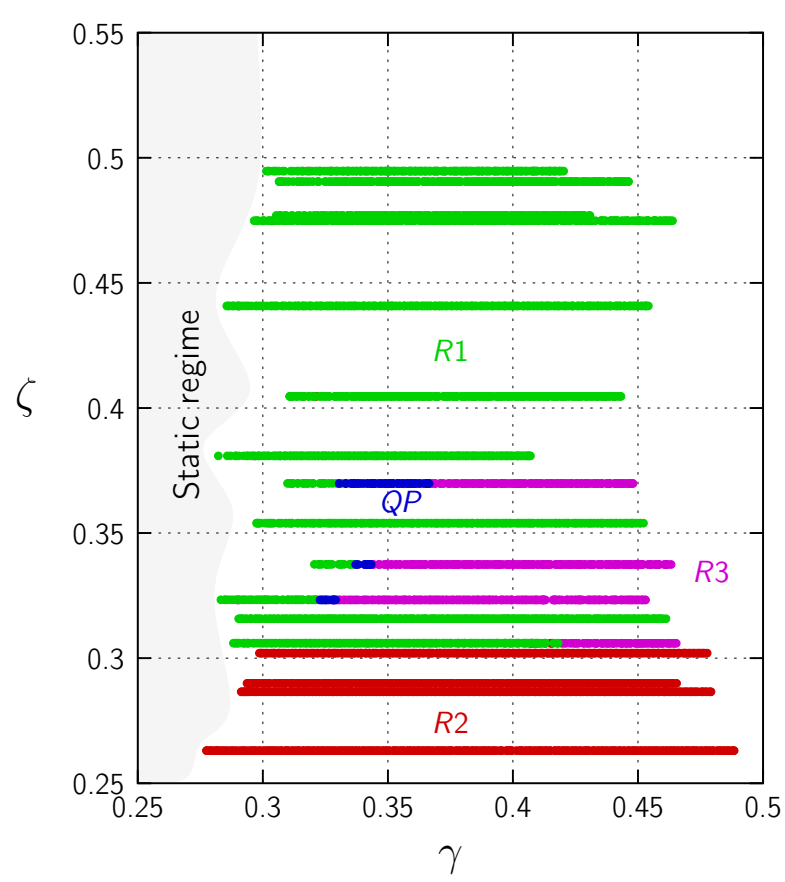

(b)

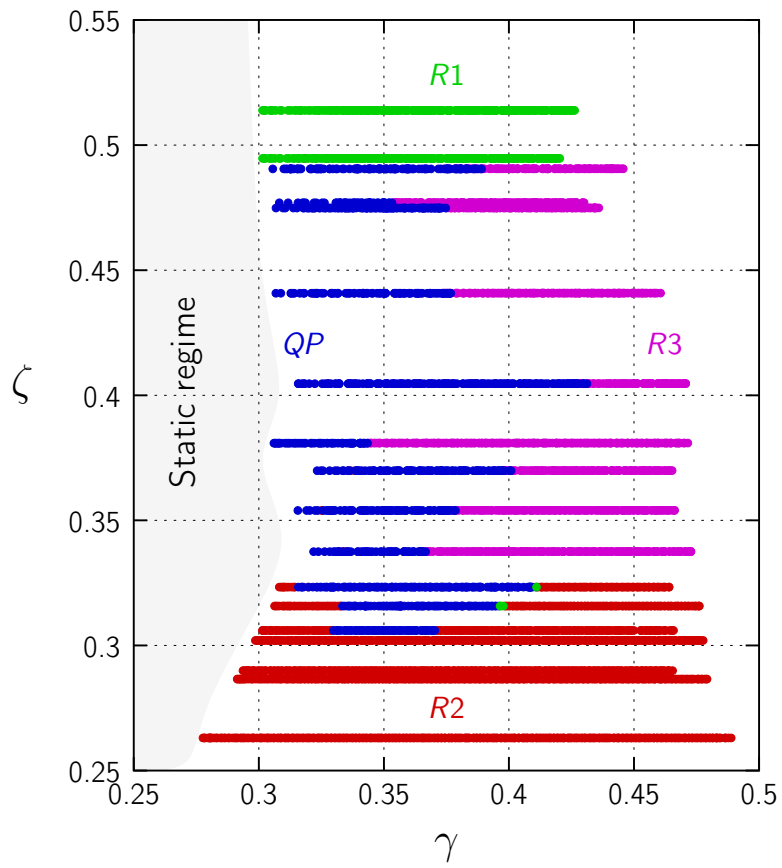

(d)

Fig. 9 - (Color online) Maps of oscillating regimes in the $(\gamma, \zeta)$ plane for different values of inharmonicity (G' fingering). (a) $\Delta f / f=105 \%$, (b) $\Delta f / f=106 \%$, (c) $\Delta f / f=108 \%$ and (d) $\Delta f / f=110 \%$. A periodic regime based on the $n^{\text {th }}$ register of the instrument is noted Rn, while QP stands for a quasi-periodic regime. Each oscillation regime is represented by a particular color. Each horizontal line corresponds to the output of the identification algorithm. 
The main conclusions of the analysis of these pictures are gathered below :

- By adjusting the control parameters, periodic oscillation regimes corresponding to the three lowest registers of the instrument are playable, except for an inharmonicity of $105 \%$ for which only R1 and R2 are played;

- Inharmonicity appears to be a key parameter for the production of quasi-periodic regimes. Indeed, above a threshold of inharmonicity (estimated around $\Delta f / f=106 \%$ ), playing quasi-periodic regimes becomes possible;

- The larger the inharmonicity, the wider the area of quasi-periodicity in the $(\zeta, \gamma)$ plane;

- In the control parameters space, quasi-periodic regimes occur at the boundary between the different periodic registers;

- The emergence of R3 or QP when inharmonicity increases comes at the expense of the playability of $\mathrm{R} 1$. Indeed, playability of R2 is nearly not altered by the increase of inharmonicity. This is not surprising since pushing/pulling the piston has only an influence on the first resonance frequency;

- From a quasi-periodic regime, it is possible to get the third (periodic) register by simply modifying the blowing pressure;

- A jump from the first register to the second requires a greater lip pressure, which corresponds to measurement on real players ${ }^{17}$.

\section{Other ways of triggering quasi-periodicity}

Maps presented so far demonstrate that the emergence of quasi-periodic regimes depends on the level of inharmonicity, but also on the values of the control parameters $(\zeta, \gamma)$. It is then interesting to investigate how quasi-periodicity emerges, for instance in the $(\gamma, \Delta f / f)$ plane, for a given value of $\zeta$. This is done in Figure 10 with $\zeta=0.37$. This map can be seen as an horizontal slice (at height $\zeta=0.37$ ) of a continuum of maps similar to those of Figure 9 . The only parameter varying through time is still the blowing pressure $\gamma$. It is worth noting that for this experiment, quasi-periodicity occurs for an inharmonicity above $\Delta f / f=106 \%$ (see Figure 10), as expected when considering Figure 9. Again, when playing crescendo through an increase of the blowing pressure, crossing through a quasi-periodic regime is required to play the third periodic register.

It is also interesting to investigate how quasiperiodicity is triggered when inharmonicity is being varied along time. The experimental device does not allow a computer control of inharmonicicy (see Section ). Thus, the piston is pushed/pulled by hand, and this is done as gently and regularly as possible. Figure 11 displays the spectrogram of the pressure signal when the variation of inharmonicity triggers the bifurcation of the periodic regime towards a quasi-periodic regime $(\gamma=0.35$ and $\zeta=0.33$ in that experiment). The quasi-periodicity threshold (i.e., the bifurcation) is estimated at $\Delta f / f=107 \%$. Before

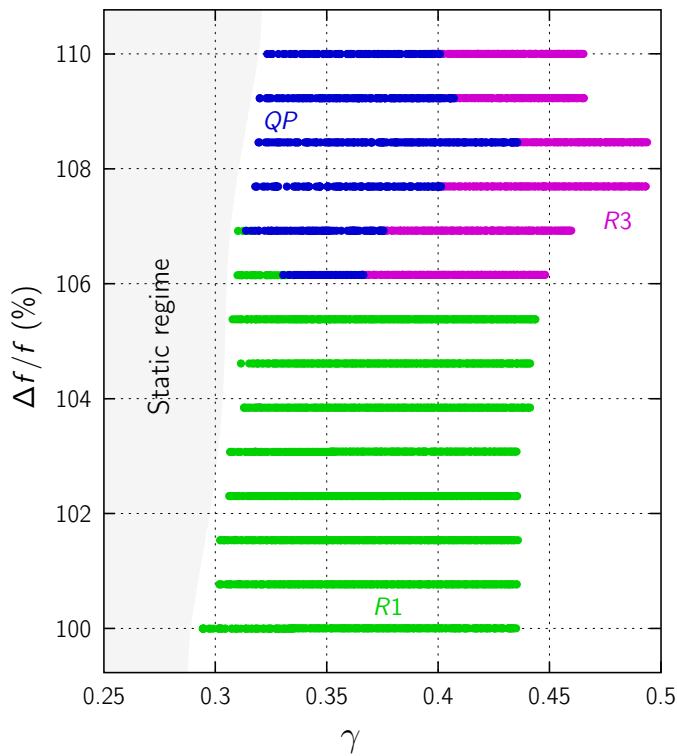

Fig. 10 - (Color online) Map of oscillating regimes in the $(\gamma, \Delta f / f)$ plane corresponding to the setting $\zeta=0.37$ ( $\mathrm{G}$ ' fingering). A periodic regime based on the $n^{\text {th }}$ register of the instrument is noted $\mathrm{R} n$, while $\mathrm{QP}$ stands for a quasi-periodic regime.

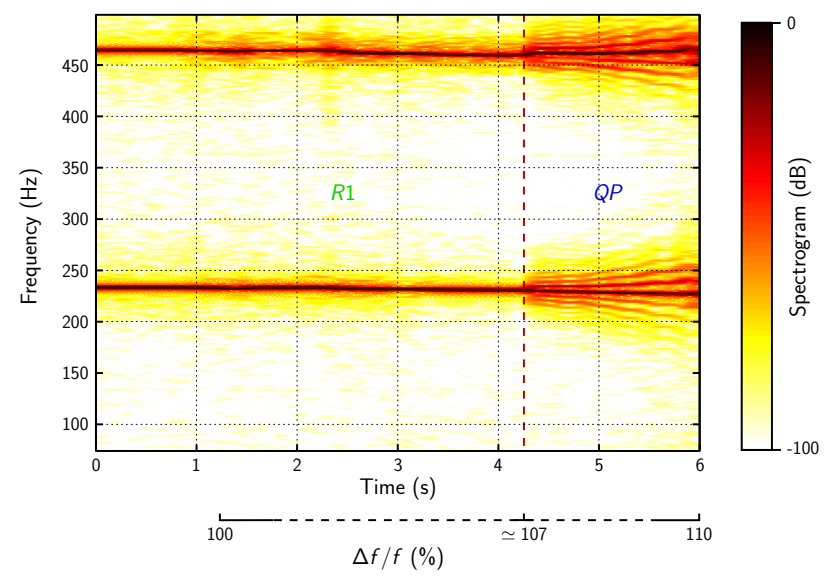

Fig. 11 - (Color online) Spectrogram of the mouthpiece pressure signal with a time-varying inharmonicity $\left(\mathrm{G}^{\prime}\right.$ fingering, $\gamma=0.35, \zeta=0.33)$.

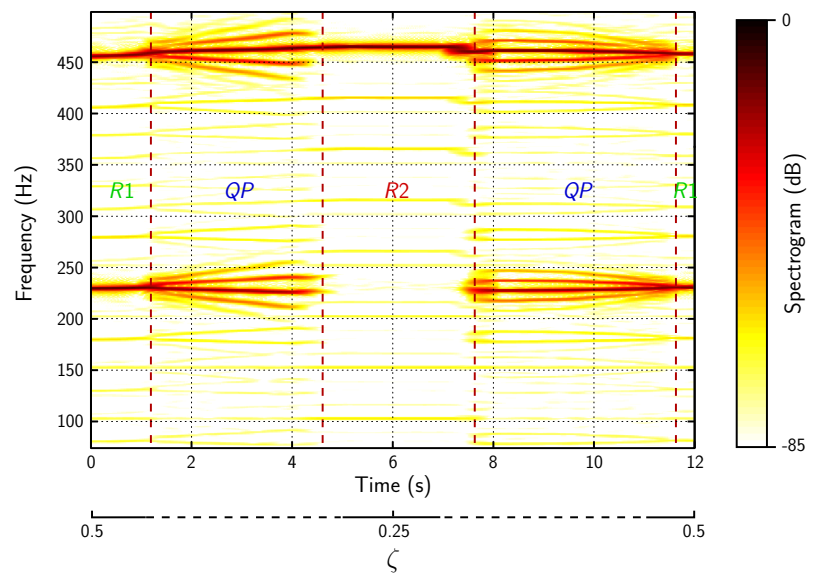

Fig. 12 - (Color online) Spectrogram of the mouthpiece pressure signal with a time-varying embouchure parameter $\zeta$ (G' fingering, $\gamma=0.35, \Delta f / f=110 \%$ ). 
the bifurcation, the inharmonicity increase (through the decrease of the lowest resonance frequency, see Figure 4) causes a decrease in the playing frequency of the first periodic regime $R 1$. This corresponds to mode locking with the first mode ${ }^{19}$. Beyond the bifurcation, the inharmonicity increase causes a rise of the modulation frequency $f_{M}$, which can be seen in the frequency domain as the increase of the gap between the spectral components of frequencies $\pm n f_{1} \pm m f_{2}$.

In a similar way, quasi-periodicity can also be triggered when the embouchure parameter $\zeta$ varies. For this purpose, the water-filled syringe which allows to modify this parameter is pushed/pulled as gently and regularly as possible. Two measurements of the NLC are carried out, at the beginning and at the end of the variation of the syringe position. This allows defining the range of $\zeta$ to be explored.

As shown in Figure 12, decreasing $\zeta$ allows to undergo a first bifurcation from the first periodic register $\mathrm{R} 1$ to a quasi-periodic regime $\mathrm{QP}$, and then a second bifurcation from the quasi-periodic regime to the second periodic register R2. The inverse sequence of bifurcations is observed when $\zeta$ decreases. It is also obvious from Figure 12 that the modification of the embouchure parameter $\zeta$ allows increasing or decreasing the modulation frequency $f_{M}$.

\section{Practical musical illustrations}

The previous results have been obtained for the G' fingering, which is imposed by the additional piston (the position of which on the neck of the saxophone is calculated according to the input impedance of this fingering). However, in order to enlarge the scope of the study, other fingerings are considered in this section. The piston is not used anymore and the inharmonicity is fixed by the instrument and estimated according to the measurement of its input impedance.

A first example is based on the low $\mathrm{C}$ fingering (noted $\mathrm{C}$ ), where nearly all lateral holes are closed. In this case, the inharmonicity of the instrument is $\Delta f / f=$ $103 \%$ (deduced from the input impedance measurement, the modulus of which is displayed in Figure 13).

For this fingering, Figure 14 displays three pressure signals measured inside the mouthpiece for different values of the embouchure parameter $\zeta$ (the blowing pressure is fixed). Figures $14(\mathrm{a})$ and 14(b) show periodic signals corresponding to the first and second register of the instrument respectively. Figure 14(c) displays a quasi-periodic signal obtained for another value of the embouchure parameter $(\zeta=0.76)$.

These measurements highlight the influence of the peaks amplitude on the oscillation regimes selection. The second register is obtained for all $\zeta<0.74$ rather than $\zeta<0.33$ for the G' fingering (when inharmonicity is at the lowest : see Fig. 9(a), and where the amplitude of the second peak is lower than the amplitude of the first : see Fig. 4). Also, quasi-periodic regimes can be produced even for low inharmonicity $(\Delta f / f=103 \%)$. Compared to the quasi-periodicity threshold for the $\mathrm{G}$ ' fingering $(\Delta f / f=106 \%)$, the amplitude rise of the second resonance mode (see Fig. 13) appears to favor

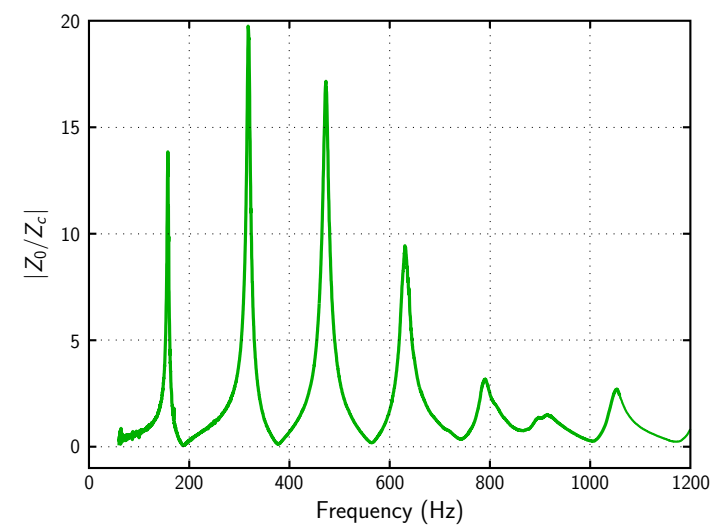

Fig. 13 - Modulus of the dimensionless input impedance of an alto saxophone (low $\mathrm{C}$ fingering, $\Delta f / f=103 \%$ ).

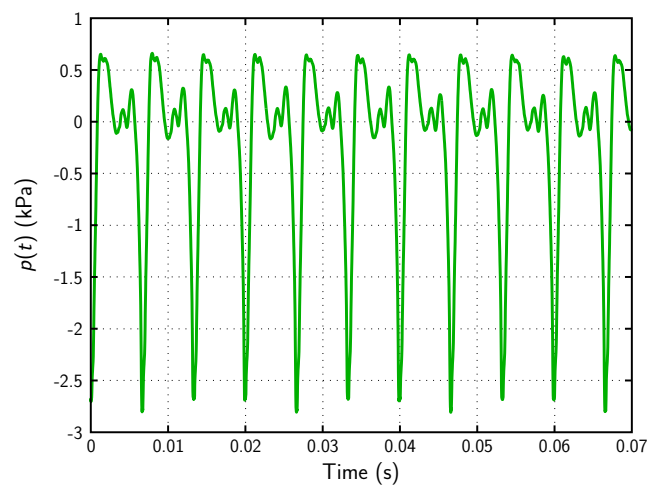

(a) First periodic register $(\zeta=0.98)$

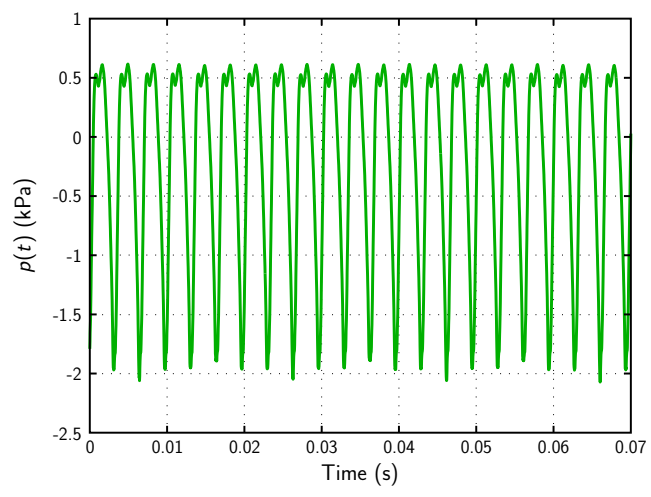

(b) Second periodic register $(\zeta=0.74)$

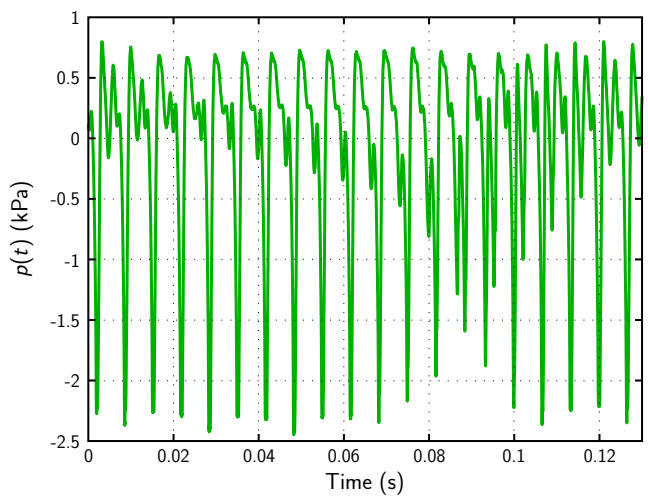

(c) Quasi-periodic regime $(\zeta=0.76)$

Fig. 14 - Mouthpiece pressure signals for different values of the embouchure parameter $\zeta$ (low $\mathrm{C}$ fingering,

$$
\gamma=0.42) \text {. }
$$


the emergence of quasi-periodicity.

A second example is based on the use of a fork fingering. This consists in playing a low $\mathrm{C}$ with one key hole open (right hand forefinger). This particular fingering is often used to play multiphonics. Figure 15 displays the corresponding modulus of the input impedance. It appears that an intermediate, low-amplitude peak, emerges between the main two lowest peaks. This is commonly observed for fork fingerings ${ }^{19}$. Inharmonicity is estimated according to the frequency of the two main peaks and reaches $\Delta f / f=115 \%$.

The map of the oscillation regimes with respect to the control parameters $(\zeta, \gamma)$ is presented in Figure 16 . It is worth noting that the range of the parameter $\zeta$ is as wide as possible experimentally. This map highlights that playing quasi-periodic sounds is possible on a wide range of the $\zeta$ parameter. For this fingering, the inharmonicity is high enough to ease the emergence of quasi-periodic regimes. However the location of these regimes regarding the blowing pressure $\gamma$ varies significantly with the value of $\zeta$.

On the one hand, for $\zeta>0.7$, the map shows that quasi-periodic oscillations are often produced between periodic registers 1 and 2 when blowing harder. This is similar to what has been seen above. On the other hand, for $\zeta<0.7$, describing the map is more complicated. This shows that the fork fingering makes it very difficult to produce oscillation regimes stable on a wide range of control parameters. This is all the more obvious when compared to a regular fingering, as seen in Figure 9(a).

This is likely why beginners experience difficulties in selecting a particular regime when playing unconventional fingerings.

\section{Discussion and conclusion}

Results presented above confirm the observation of the $\operatorname{article}^{10}$ : inharmonicity is a necessary condition in the production of quasi-periodic regimes with saxophones. However it is not a sufficient condition since the two control parameters which were tested, proved to have a significant influence on the triggering of quasi-periodicity. Complementing what has been stated in article ${ }^{10}$, the control parameters also influence the beating frequency of quasi-periodic oscillations. Indeed, as shown in the figures 11 and 12, the change of control parameters can strongly modify the modulation frequency $f_{M}$ (even if the inharmonicity is constant).

The trends highlighted experimentally in this article are in agreement with the numerical results obtained in a previous work ${ }^{11}$. This numerical study was based on the use of a minimal model of wind instruments, so it is possible to deduce that features neglected in this model are not determining in the production of quasi-periodic regimes (inertia and damping of the reed, resonance modes higher than the third one).

Compared to articles ${ }^{10 ; 11}$, experiments presented in this article highlight other trends in the oscillation regimes production. When inharmonicity is high enough, the production of higher register (unplayable otherwise) is favoured. Also by testing several fingerings, the amplitude of resonance modes appears to be a determining

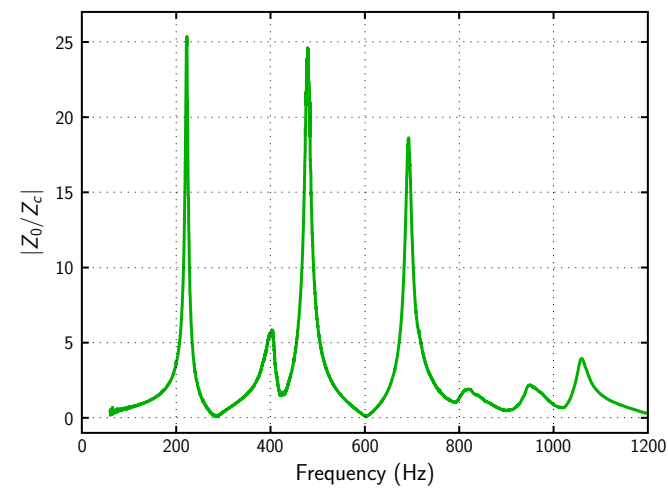

Fig. 15 - Modulus of the dimensionless input impedance of an alto saxophone (fork fingering, $\Delta f / f=115 \%$ ).

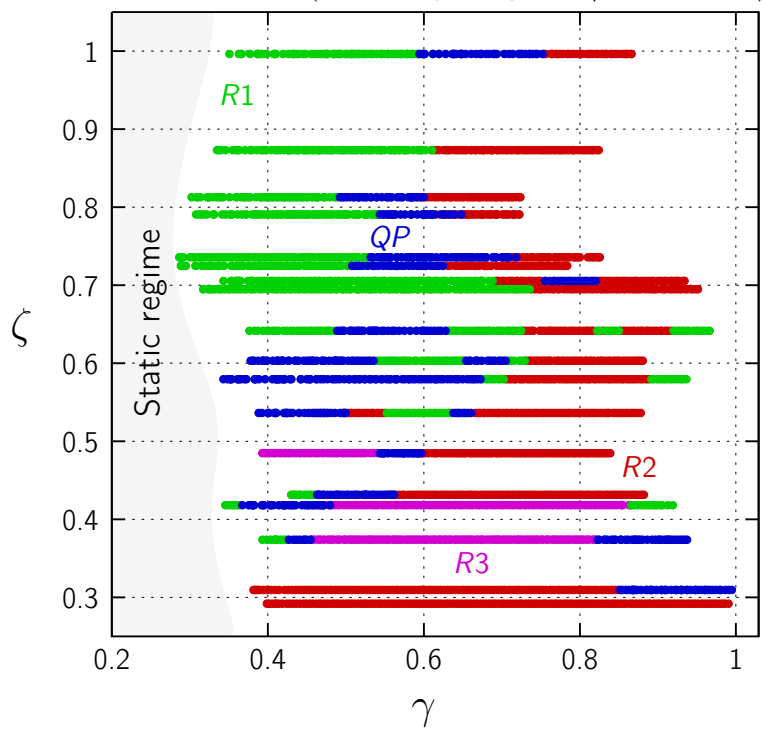

Fig. 16 - (Color online) Map of oscillating regimes in the $(\gamma, \zeta)$ plane for a fork fingering. A periodic regime based on the $n^{\text {th }}$ register of the instrument is noted $\mathrm{R} n$, while QP stands for a quasi-periodic regime.

factor in playability of registers.

All trends presented in this article are qualitative. This was intended to globally describe how the instrument can produce different oscillation regimes. Many experimental parameters can alter the bifurcation thresholds of the various registers (speed variation of the supply pressure, reed force, composition of artificial reeds, use of vocal-tract support, etc.). Therefore, an interesting work would consist in extending this experimental study in order to obtain more quantitative results. This would allow to study the influence of the control parameters on the bifurcation thresholds of reed instruments.

\section{Acknowledgements}

The authors wish to thank Joël Gilbert for his precious advice as well as for the loan of the crook used for the experiments carried out in this article. Also, the authors wish to acknowledge Jean Kergomard for his valuable advice during the preparation of this manuscript. This work has been done in the framework of Labex MEX (ANR-10-LABX-0092) and of the project A*MIDEX (ANR-11-IDEX-0001-02), funded by the French National Research Agency (ANR).

\section{REFERENCES}


1. N. H. Fletcher and H. D. Rossing, The physics of musical instruments (Springer-Verlag, 2th edition, 1998), chapter 15, pp. 477-480.

2. A. H. Benade, Fundamentals of musical acoustics (Oxford University Press, 1976), chapter 25.

3. V. Gibiat, "Phase space representations of acoustical musical signals," J. Acoust. Soc. Am. 123(3), 529-536 (1988).

4. J. Backus, "Multiphonic tones in the woodwind instruments," J. Acoust. Soc. Am. 63(2), 591-599 (1978).

5. N. H. Fletcher, "Sound production by organ flue pipes," J. Acoust. Soc. Am. 60(4), 926-936 (1976).

6. N. H. Fletcher and L. M. Douglas, "Harmonic generation in organ pipes, recorders, and flutes," J. Acoust. Soc. Am. 68(3), 767-771 (1980).

7. J. W. Coltman, "Jet offset, harmonic content, and warble in the flute," J. Acoust. Soc. Am. 120(4), 2312-2319 (2006).

8. D. H. Keefe and B. Laden, "Correlation dimension of woodwind multiphonic tones," J. Acoust. Soc. Am. 90(4), 1754-1765 (1991).

9. T. Idogawa, T. Kobata, K. Komuro and M. Iwaki, "Nonlinear vibrations in the air column of a clarinet artificially blown," J. Acoust. Soc. Am. 93(1), 540-551 (1993).

10. J.-P. Dalmont, B. Gazengel, J. Gilbert and J. Kergomard, "Some aspects of tuning and clean intonation in reed instruments," Appl. Acoust. 46(1), 19-60 (1995).

11. J.-B. Doc, C. Vergez and S. Missoum, "A minimal model of a single-reed instrument producing quasi-periodic sounds," Acta Acust. Acust. 100(3), 543-554 (2014).

12. A. Almeida, D. George, J. Smith and J. Wolfe, "The clarinet : How blowing pressure, lip force, lip position and reed "hardness" affect pitch, sound level, and spectrum," J. Acoust. Soc. Am. 134(3), 2247-2255 (2013).

13. D. Ferrand, Ch. Vergez, B. Fabre and F. Blanc, "High-precision regulation of a pressure controlled artificial mouth : The case of recorder-like musical instruments," Acta Acust. Acust. 96(4), 701-712 (2010).

14. A. Chaigne and J. Kergomard, Acoustics of musical instruments (English Translation, Belin, 2008), pp. 413-416.

15. J.-P. Dalmont, J. Gilbert and S. Ollivier, "Nonlinear characteristics of single-reed instruments : Quasistatic volume flow and reed opening measurements," J. Acoust. Soc. Am. 114(4), 2253-2262 (2003).

16. C. A. Macaluso and J.-P. Dalmont, "Trumpt with near perfect harmonicity : Desgin and acoustic results," J. Acoust. Soc. Am. 129(1), 404-414 (2011).

17. Ph. Guillemain, C. Vergez, D. Ferrand and A. Farcy, "An instrumented saxophone mouthpiece and its use to understand how an experienced musician plays," Acta Acust. Acust. 96(4), 622-634 (2010).
18. J.-P. Dalmont, J. Gilbert and J. Kergomard, "Reed instruments, from small to large amplitude periodic oscillations and the helmholtz motion analogy," Acta Acust. Acust. 86(4), 671-684 (2000).

19. C. J. Nederveen and J.-P. Dalmont, "Mode locking effects on the playing frequency for fork fingerings on the clarinet," J. Acoust. Soc. Am. 131, 689-697 (2012). 